\title{
Strategies to Improve Persistent Adherence in Colorectal Cancer Screening
}

\author{
Guan Sen Kew ${ }^{1}$ and Calvin Jianyi Koh ${ }^{1,2}$ \\ ${ }^{1}$ Division of Gastroenterology and Hepatology, Department of Medicine, National University Hospital, and ${ }^{2}$ Department of Medicine, Yong Loo \\ Lin School of Medicine, National University of Singapore, Singapore
}

Colorectal cancer is a major cause of morbidity and mortality, and screening has been shown to reduce morbidity and mortality. This review highlights pertinent aspects of adherence to screening including the various options available and key concepts to consider in any systematic program. Persistent adherence, which is key to an efficacious screening program, is the compliance with repeated screening tests over a period of time. A "chain of survival" mindset emphasizes the stepwise, sequential and persistent approach to screening and is a helpful concept to drive the message of persistent adherence. A framework for the patient and physician interactions that support screening intervention is examined, and various factors that impact both patient and physician adoption and screening modality recommendations are reviewed. While systems-based approaches to screening have great utility in automation and monitoring in a surveillance program, some emerging data suggests that the human touch is still an essential driver for active participation in these programs. Finally, the proportion of time concept is discussed as a useful index for monitoring persistent adherence in both individuals and populations. (Gut Liver 2020;14:546-552)

Key Words: Colorectal neoplasms; Screening; Adherence; Strategies; Chain of survival

\section{INTRODUCTION}

Colorectal cancer (CRC) is an important public health problem and is a major cause of morbidity and mortality. It is the third most common cancer diagnosed and the fourth cause of cancerrelated deaths worldwide. ${ }^{1}$ According to the International Agency for Research on Cancer, there were 1,849,518 new cases and 880,792 cancer deaths in 2018. ${ }^{2}$ Approximately 4.6\% of men (1 in 22 ) and $4.2 \%$ of women (1 in 24) will be diagnosed with CRC in their lifetime. ${ }^{3}$
Most international guidelines recommend regular CRC screening in average-risk adults aged 50 years and older ${ }^{4,5}$ and options for screening include stool-based tests (fecal immunochemical test [FIT], high sensitivity guaiac-based fecal occult blood test [FOBT] or multitarget stool DNA test) or structural examinations (colonoscopy, computed tomography [CT] colonography, or flexible sigmoidoscopy). However, despite strong evidence that CRC screening has unequivocal mortality benefit, ${ }^{6-8}$ the uptake of screening tests remains low, with an estimated adherence rate of $13 \%$ to $55 \%$ based on community surveys done in the United States. ${ }^{9}$ The adherence rates to screening tests are no better in Asia-Pacific countries, ranging from 21\% in South Korea to $62.9 \%$ in Thailand based on a review article by Navarro et al. ${ }^{10}$ Given that survival is strongly related to stage at diagnosis and given the benefit of CRC screening, it is important to evaluate and improve adherence to CRC screening in our communities.

This review article aims to examine the various factors limiting adherence, and strategies we can employ to improve adherence in CRC screening.

\section{DEFINITION}

Adherence is defined as the proportion of target population compliant with the current recommendations on CRC screening/ surveillance, and in other words-the rates for patients that were offered screening and got screened.

Persistent adherence is the compliance to repeated screening tests over a period of time, and is generally harder to measure, given that various CRC screening modalities have different recommended intervals for repeat screening and surveillance. ${ }^{11}$ It is persistent adherence, rather than once-off adherence to a CRC screening modality, that is key to program efficacy and screening effectiveness, particularly for fecal-based screening tests where repeated testing improves performance.

\section{Correspondence to: Calvin Jianyi Koh}

Division of Gastroenterology and Hepatology, Department of Medicine, National University Hospital, 1E Kent Ridge Rd, Singapore 119228, Singapore Tel: +65-6772-5555, Fax: +65-9775-1518, E-mail: calvin_j_koh@nuhs.edu.sg

Received on September 13, 2019. Revised on October 22, 2019. Accepted on October 31, 2019. Published online December 13, 2019. pISSN 1976-2283 eISSN 2005-1212 https://doi.org/10.5009/gnl19306

@ This is an Open Access article distributed under the terms of the Creative Commons Attribution Non-Commercial License (http://creativecommons.org/licenses/by-nc/4.0) which permits unrestricted non-commercial use, distribution, and reproduction in any medium, provided the original work is properly cited. 


\section{CRC SCREENING CONTINUUM}

For any screening to occur, there needs to be a sequential, stepwise series of coordinated events that occur between the target population, the primary care provider, and second-order services. This is akin to coordinating a series of tasks in resuscitation for a cardiac arrest (activation of the emergency response system, cardiopulmonary resuscitation, defibrillation, basic and advanced emergency medical services and advanced life support). Taking a leaf from the chain of survival in resuscitation of cardiac arrest patients by the American Heart Association, ${ }^{12}$ we propose a CRC screening continuum (see Fig. 1) in our target population, where the healthy patient will have to go through various stages in CRC screening from being a healthy patient, to a screening healthcare encounter, then the screening modality, followed by receiving further treatment based on the outcome of the screening modality. However, unlikely the chain of survival, the CRC screening continuum forms a closed loop, and the patient would have to undergo same cycle again for repeat screening and surveillance, with the interval dependent on the type of screening modality and outcome of the screening test.

In reality, many patients do not progress beyond the initial screening, especially with fecal-based screening tests which require yearly screening to improve its efficacy. This lack of feedback loop with the healthcare system after the screening test might contribute to the challenges in engaging the patient in screening persistence. In a retrospective cohort analysis performed by Liss et al., ${ }^{13}$ they found that only $24.6 \%$ of the initial population who had completed a FOBT, went on to complete a second FOBT between 9 to 18 months. The low persistent adherence rate to FOBT was further supported by another study by Fenton et al., ${ }^{14}$ who concluded that less than half of their patients (44.4\%) completed repeated FOBT screening during the 2 year-observation period.

For both providers as well as patients, having a "chain of survival" mindset toward screening will change the paradigm from screening being a once-off event, to something that needs to be sustained and adhered to at a regular interval. Cancer screening is more of the marathon than a sprint.

\section{FACTORS AFFECTING ADHERENCE}

Understanding the factors that influence adherence to CRC screening is imperative before we can embark on strategies to optimize adherence rates and encourage better screening participation. These factors can be broadly classified to non-modifiable (age, gender, race, marital status, education level, and family history of CRC) and modifiable (health seeking behavior, attitudes and knowledge about (RC).

In evaluating adherence with screening guidelines, one can examine both patient and physician attitudes and interactions, as illustrated in Fig. 2. Subramanian et al. ${ }^{9}$ proposed a theoretical framework which illustrates the dynamic relationship between physician recommendation and patient adherence. They highlighted factors impacting patient adherence including physician recommendation, patient demographics, financial enablers, perceived CRC risk and health care system interactions. On the other hand, physician recommendations are in turn influenced by patient compliance, perceived test effectiveness, physician demographics, guideline awareness and health system factors.

This is a helpful conceptual framework as it recognizes both physician and patient factors and the complex interplay of the physician-patient relationship in screening recommendation and adherence.

Similar patient factors limiting adherence were also found in a Taiwanese study involving a cross-sectional telephone survey, ${ }^{15}$ which included 2,807 study participants, and the following factors were found to be associated with undergoing a confirmatory colonoscopy after a positive FIT-young age, being married, higher perceived threat, higher cue for action (such as physician recommendation), and higher health behavior scores.

The type of screening modality also has an impact on adherence rate, and in general less invasive tests have a higher uptake rate. In the COLONPREV Study by Castells and Quintero, ${ }^{16}$ 57,404 individuals were randomized to receive either colonoscopy or FIT, and the authors found that the overall participation rate was $25.0 \%$ in the colonoscopy arm, and 34.2\% in the FIT group (odds ratio [OR], 0.63; 95\% confidence interval [CI], 0.60

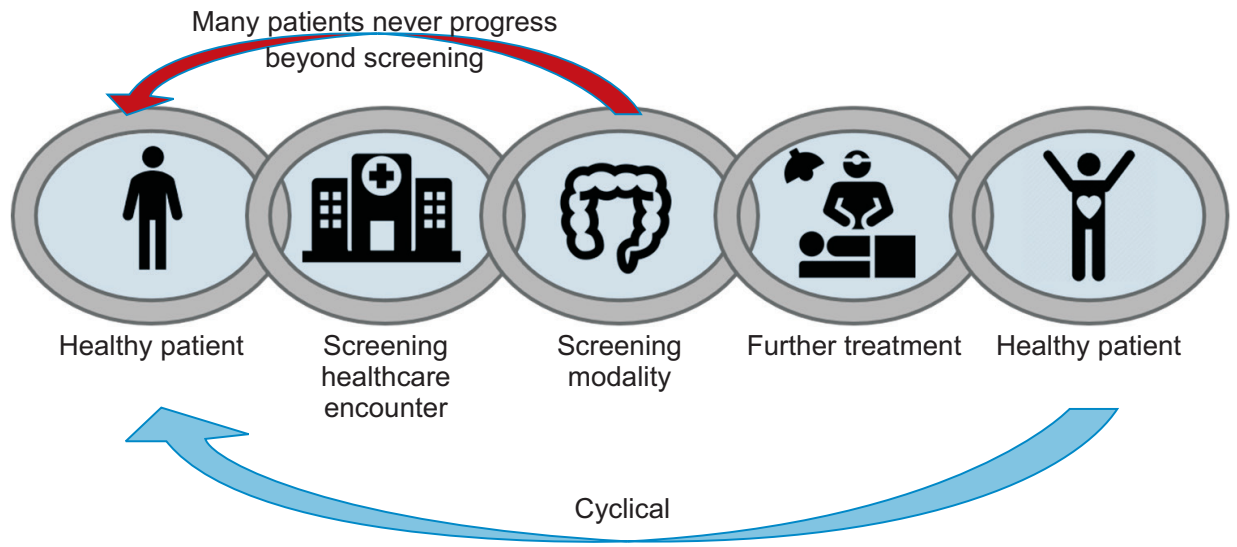

Fig. 1. Colorectal cancer screening continuum. 


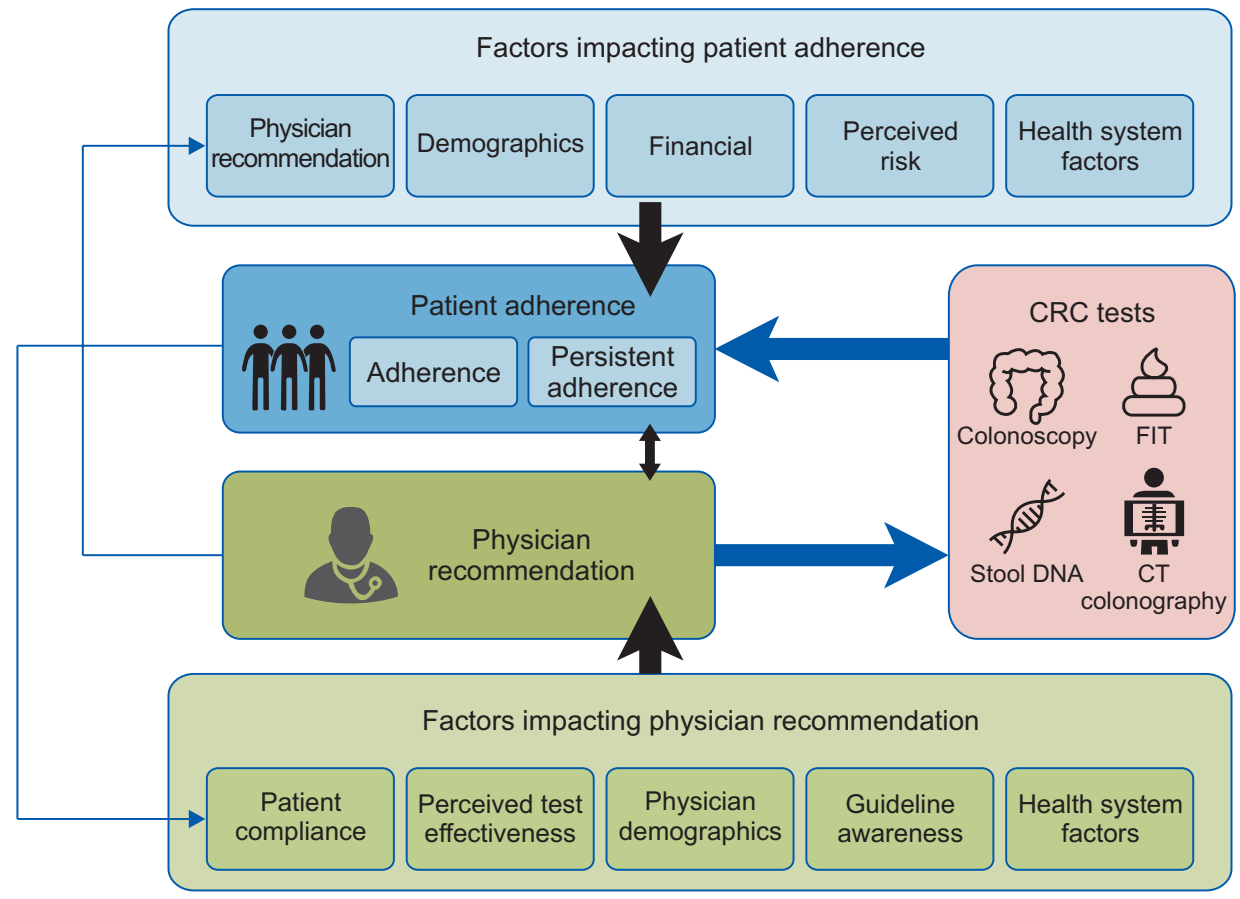

Fig. 2. Factors affecting adherence. CRC, colorectal cancer; FIT, fecal immunochemical test; CT, computed tomography. Adapted from Subramanian S, et al. Prev Med 2004;38:536-550.

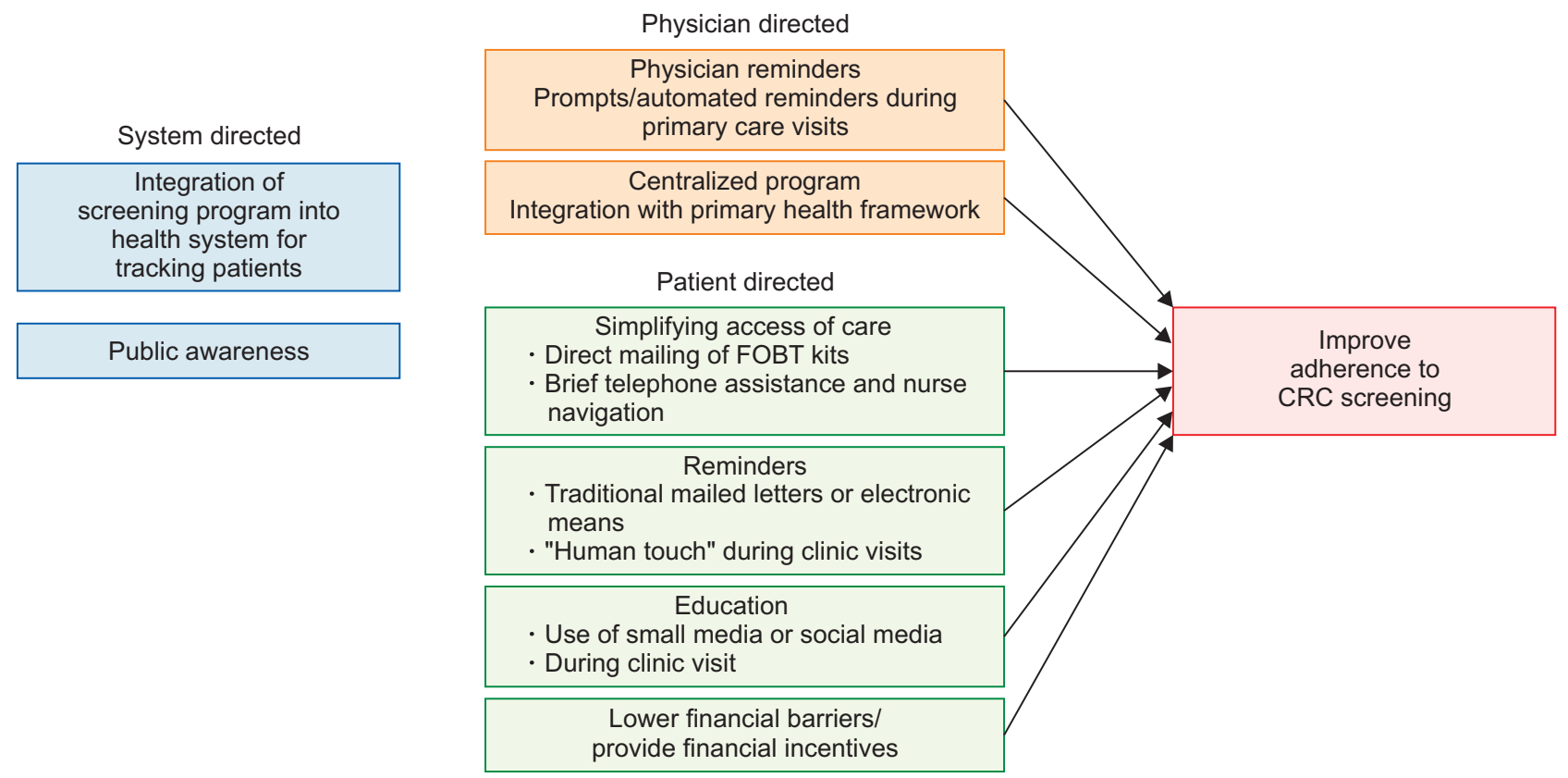

Fig. 3. Strategies to improve adherence to colorectal cancer (CRC) screening. FOBT, fecal occult blood test.

to 0.65 ; $\mathrm{p}<0.001)$. Similarly, in a meta-analysis comparing colonoscopy with CT colonography, the study found that the participation rate for colonoscopy was much lower at 20.0\%, compared to $29.0 \%$ for CT colonography. ${ }^{17}$ When given the choice, patients generally prefer a noninvasive modality, but this differs greatly from the investigations that patients actually undergo in most units, suggesting that clinicians play a significant role in modifying patients' choice. ${ }^{18}$

\section{STRATEGIES TO IMPROVE ADHERENCE}

Promoting CRC screening and surveillance in any population involves a system-based and holistic approach, and would require a rigorous health policy that is put in place to encourage adherence. This would involve educating and engaging the public, having a system in place to track patients who are overdue for screening, sending reminders to participants and simplifying 
Patient A: 2 years $(20.0 \%)$ covered

\begin{tabular}{|l|l|l|l|l|}
\hline & & & & \\
\hline $\begin{array}{l}\text { Cohort entry: } \\
\text { January 1, 2009 }\end{array}$ & $\begin{array}{l}\text { FIT, normal findings } \\
\text { July 5, 2010 }\end{array}$ & $\begin{array}{l}\text { FIT, normal findings } \\
\text { March 10, 2016 }\end{array}$ & $\begin{array}{l}\text { End of study period } \\
\text { December 31, 2018 }\end{array}$ \\
\hline
\end{tabular}

Patient B: 6 years $(60.0 \%)$ covered

\begin{tabular}{|l|l|l|l|}
\hline & & & \\
\hline $\begin{array}{l}\text { Cohort entry: } \\
\text { January 1, 2009 }\end{array}$ & $\begin{array}{l}\text { FIT, normal findings } \\
\text { January 10, 2010 }\end{array}$ & $\begin{array}{l}\text { Sigmoidoscopy, normal findings } \\
\text { February 4, 2013 }\end{array}$ & $\begin{array}{l}\text { End of study period } \\
\text { December 31, 2018 }\end{array}$
\end{tabular}

Patient C: 9 years $(90.0 \%)$ covered

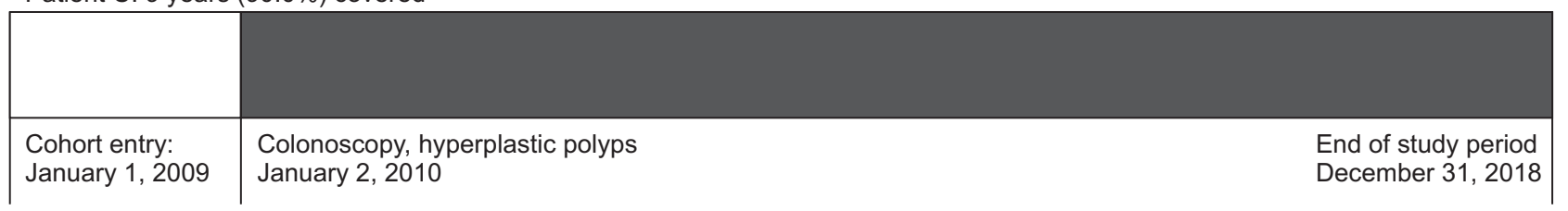

Fig. 4. Illustration of the proportion of time covered concept in a cohort followed for over 10 years.

FIT, fecal immunochemical test. Adapted from Murphy CC, et al. Gastrointest Endosc 2018;88:323-331. ${ }^{33}$

access of care to reduce provider-related barriers. In a randomized controlled trial involving 4,675 individuals conducted in Washington State, USA, a centralized program compromising of clinic-based strategies, mailed fecal tests, brief telephone assistance and nurse navigation had resulted in intervention participants having 31\% more adjusted covered time over 5 years compared to patients in the control arm who received only usual care. ${ }^{19}$ This demonstrated the importance and effectiveness of a centralized program and framework to improve CRC participation rates.

Raising public awareness on CRC and changing their attitudes and beliefs is critical in engaging the local population on this major health problem. This can be carried out either via the use of small media such as informational or educational material delivered in pamphlets, brochures, newsletters, television and social media, or opportunistically during clinic visits with one's primary healthcare provider. Studies such as those performed by Church et al., ${ }^{20}$ Denberg et al., ${ }^{21}$ and Wardle et al. ${ }^{22}$ found that participation rates in CRC screening were higher after educational brochures were mailed to the study participants. Patients who are overdue for screening may be identified by their primary or community healthcare providers and reminders can be sent to these individuals either via electronic means or traditional mailed letters.

Reduction of structural barriers to CRC screening is usually achieved via direct mailing of FOBT kits with or without prepaid postage, and although this strategy may be expensive, there are a number of studies ${ }^{23-25}$ that have shown that it is useful in increasing CRC screening participation rate. In a study comparing usual care versus mailed FOBT with instructions, the authors

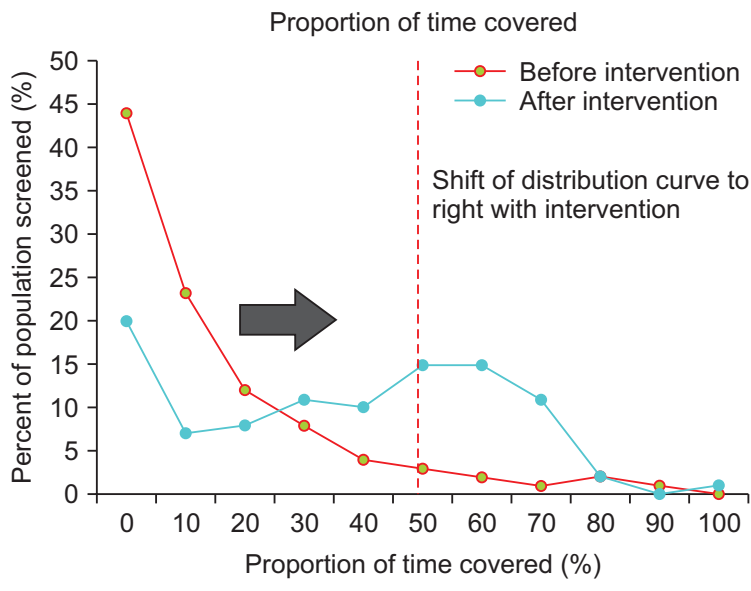

Fig. 5. The proportion of time before and after intervention. Illus trating the effect of a population intervention on the proportion of time covered metric-post intervention, more of the population lies to the right of the arbitrary cutoff, in this case 50\% proportion of time screened.

found that participation rate was only a mere $2 \%$ in the usual care group versus $26 \%$ in the FOBT group. ${ }^{26}$ The "human touch" also plays an important role in promoting adherence as seen in a large French study, in which participants were more likely to participate if they received the FOBT kit from their private general practitioner compared to if they were to receive the FOBT kit via mail, with an OR of 10.7 (95\% CI, 10.0 to 11.5$)^{27}$

Personalization and practical measures work not just on patients, but also on physicians. Rat et al. ${ }^{28}$ evaluated general practitioners on the west coast of France, and showed that providing a specific list of patients who were not up to date with 
their screening had a statistically significant increase of about 3\% over generic reminders about the regional screening rate, and no reminders at all. Although resource intensive in approach, the use of computer databases and automation should allow this strategy to be broadly adopted in digitally-enabled health systems.

Offering financial incentives to promote compliance to CRC screening, has not been shown to be useful in increasing participation rate, as demonstrated in a study by Gupta et al., ${ }^{29}$ in which the low-income, uninsured population, when given a financial incentive of either $\$ 5$ or $\$ 10$ had not resulted in a higher completion FIT rate.

A summary of strategies to improve adherence to CRC screening is illustrated in Fig. 3.

\section{FUTURE TRENDS IN CRC SCREENING}

In recent years, there has been an emerging trend that bloodbased biomarkers have the potential as a diagnostic and prognostic tool in CRC. ${ }^{30}$ Compared to current modalities of CRC screening such as fecal-based tests or colonoscopy, serum biomarkers are noninvasive and convenient, with the potential to improve patients' compliance to CRC screening. The most wellknown CRC biomarker is the methylated SEPT9 DNA test, which had an overall sensitivity of $56.1 \%$ to $79.3 \%$ with a specificity of $89.6 \%$ to $99.1 \%$ based on 13 studies which had attempted to evaluate this assay. ${ }^{31}$ However, commercial tests based on SEPT9 methylation are more expensive compared to stool-based tests, and are not widely available, hence limiting the utilization of such assays.

Other noninvasive blood tests targeting various molecular markers appear to be on the horizon. In a recent study by Cohen et al., ${ }^{32}$ the authors describe a blood test called CancerSEEK to detect eight common cancer types through assessment of the levels of circulating proteins and mutations in cell-free DNA. They applied this test to 1,005 patients, with non-metastatic, clinically detected cancers of the ovary, liver, stomach, pancreas, esophagus, colorectum, lung or breast. The median sensitivity of CancerSEEK among the eight cancer types evaluated was $70 \%$, and ranged from 33\% in breast cancers to 98\% in ovarian cancers, with a specificity of more than $99 \%$. The study lays the foundation for a single multi-analyte blood test for cancers of many types, but its clinical utility and benefit have to be firmly established in population-based prospective studies before wider adoption.

Measuring screening adherence over time is challenging and a novel way to measure adherence had emerged recently-using the proportion of time covered (PTC) approach, as suggested in a study by Murphy et al. ${ }^{33}$ Rather than once-off measurement of adherence, it provides a visual and informative estimate of screening adherence over an extended time period, identifying frequent coverage gaps and screening failures at clinically rele- vant time points. The assigned time covered is dependent on the examination type, quality and findings. For example, a normal FIT, sigmoidoscopy and colonoscopy will give a coverage period of 1 year, 5 years and 10 years respectively. An illustration of the PTC concept is seen in Fig. 4, showing in the first part how the proportion of time is calculated for three patients. Patient A with two negative, spaced-apart FITs over 10 years, would have a PTC of 20\%, patient B, has a negative FIT and then flexible sigmoidoscopy a year later, would have coverage of $60 \%$ and patient $\mathrm{C}$ who on second year of enrollment undergoes a full colonoscopy only detecting small hyperplastic polyps would be covered in the 10-year period for 90\%.

These numbers can then assessed on a cohort level which is illustrated in Fig. 5, where a hypothetical population undergoes an intervention (e.g., automated notification of screening eligibility). The intended result as shown as a shift of the PTC curve to the right, as a higher percentage of the population has a higher PTC. For example, in Fig. 4, prior to intervention the redline shows only $9 \%$ of the population attained the PTC threshold of $50 \%$, whereas after intervention $44 \%$ of the population has attained this threshold. While not as intuitive as the traditional percentage-screened metric, this measurement is able to summarize both individual as well as population-level adherence to screening programs, and demonstrate the efficacy of various population-based interventions.

\section{CONCLUSION}

High persistent adherence is key to any screening program, and it is important to recognize the factors affecting adherence so that we can implement specific interventions to target these barriers. A systems-based approach in education and facilitation, together with a comprehensive central screening program will help to promote adherence. Persistent adherence, rather than the once-off compliance to screening that we should aim to achieve, and this can be better illustrated and studied using the PTC metric proposed..$^{33}$ Less-invasive screening tools on the horizon might be a paradigm shift by lowering barriers to compliance, but as the adage goes, the best test is the test that is actually done. Many challenges still lie ahead in achieving and maintaining high adherence rates to screening.

\section{CONFLICTS OF INTEREST}

No potential conflict of interest relevant to this article was reported.

\section{ORCID}

Guan Sen Kew

Calvin Jianyi Koh https://orcid.org/0000-0002-0892-9311

https://orcid.org/0000-0002-1756-3737 


\section{REFERENCES}

1. Araghi M, Soerjomataram I, Jenkins M, et al. Global trends in colorectal cancer mortality: projections to the year 2035. Int J Cancer 2019;144:2992-3000.

2. International Agency for Research on Cancer (IARC). Colorectal cancer fact sheet [Internet]. Lyon: LARC; c2018 [cited 2019 Nov 12]. Available from: http://gco.iarc.fr/today/data/factsheets/ cancers/10_8_9-Colorectum-fact-sheet.pdf.

3. Siegel RL, Miller KD, Jemal A. Cancer statistics, 2016. CA Cancer J Clin 2016;66:7-30

4. Sung JJ, Ng SC, Chan FK, et al. An updated Asia Pacific Consensus Recommendations on colorectal cancer screening. Gut 2015;64:121-132.

5. US Preventive Services Task Force, Bibbins-Domingo K, Grossman DC, et al. Screening for colorectal cancer: US Preventive Services Task Force recommendation statement. JAMA 2016;315:25642575.

6. Kahi CJ, Imperiale TF, Juliar BE, Rex DK. Effect of screening colonoscopy on colorectal cancer incidence and mortality. Clin Gastroenterol Hepatol 2009;7:770-775.

7. Schoen RE, Pinsky PF, Weissfeld JL, et al. Colorectal-cancer incidence and mortality with screening flexible sigmoidoscopy. N Engl J Med 2012;366:2345-2357.

8. Atkin WS, Edwards R, Kralj-Hans I, et al. Once-only flexible sigmoidoscopy screening in prevention of colorectal cancer: a multicentre randomised controlled trial. Lancet 2010;375:1624-1633.

9. Subramanian S, Klosterman M, Amonkar MM, Hunt TL. Adherence with colorectal cancer screening guidelines: a review. Prev Med 2004;38:536-550.

10. Navarro M, Nicolas A, Ferrandez A, Lanas A. Colorectal cancer population screening programs worldwide in 2016: an update. World J Gastroenterol 2017;23:3632-3642.

11. Wolf AMD, Fontham ETH, Church TR, et al. Colorectal cancer screening for average-risk adults: 2018 guideline update from the American Cancer Society. CA Cancer J Clin 2018;68:250-281.

12. Cummins RO, Ornato JP, Thies WH, Pepe PE. Improving survival from sudden cardiac arrest: the "chain of survival" concept: a statement for health professionals from the Advanced Cardiac Life Support Subcommittee and the Emergency Cardiac Care Committee, American Heart Association. Circulation 1991;83:1832-1847.

13. Liss DT, Petit-Homme A, Feinglass J, Buchanan DR, Baker DW. Adherence to repeat fecal occult blood testing in an urban community health center network. J Community Health 2013;38:829833.

14. Fenton JJ, Elmore JG, Buist DS, Reid RJ, Tancredi DJ, Baldwin LM. Longitudinal adherence with fecal occult blood test screening in community practice. Ann Fam Med 2010;8:397-401.

15. Cheng SY, Li MC, Chia SL, et al. Factors affecting compliance with confirmatory colonoscopy after a positive fecal immunochemical test in a national colorectal screening program. Cancer 2018;124:907-915
16. Castells A, Quintero E. Programmatic screening for colorectal cancer: the COLONPREV study. Dig Dis Sci 2015;60:672-680.

17. Duarte RB, Bernardo WM, Sakai CM, et al. Computed tomography colonography versus colonoscopy for the diagnosis of colorectal cancer: a systematic review and meta-analysis. Ther Clin Risk Manag 2018;14:349-360

18. Ojidu H, Palmer H, Lewandowski J, et al. Patient tolerance and acceptance of different colonic imaging modalities: an observational cohort study. Eur J Gastroenterol Hepatol 2018;30:520-525.

19. Green BB, Anderson ML, Cook AJ, et al. A centralized mailed program with stepped increases of support increases time in compliance with colorectal cancer screening guidelines over 5 years: a randomized trial. Cancer 2017;123:4472-4480.

20. Church TR, Yeazel MW, Jones RM, et al. A randomized trial of direct mailing of fecal occult blood tests to increase colorectal cancer screening. J Natl Cancer Inst 2004;96:770-780.

21. Denberg TD, Coombes JM, Byers TE, et al. Effect of a mailed brochure on appointment-keeping for screening colonoscopy: a randomized trial. Ann Intern Med 2006;145:895-900.

22. Wardle J, Williamson S, McCaffery K, et al. Increasing attendance at colorectal cancer screening: testing the efficacy of a mailed, psychoeducational intervention in a community sample of older adults. Health Psychol 2003;22:99-105.

23. Potter MB, Somkin CP, Ackerson LM, et al. The FLU-FIT program: an effective colorectal cancer screening program for high volume flu shot clinics. Am J Manag Care 2011;17:577-583.

24. Hoffman RM, Steel SR, Yee EF, et al. A system-based intervention to improve colorectal cancer screening uptake. Am J Manag Care 2011;17:49-55

25. Sequist TD, Zaslavsky AM, Marshall R, Fletcher RH, Ayanian JZ. Patient and physician reminders to promote colorectal cancer screening: a randomized controlled trial. Arch Intern Med 2009;169:364-371.

26. Coronado GD, Golovaty I, Longton G, Levy L, Jimenez R. Effectiveness of a clinic-based colorectal cancer screening promotion program for underserved Hispanics. Cancer 2011;117:1745-1754.

27. Pornet C, Denis B, Perrin P, Gendre I, Launoy G. Predictors of adherence to repeat fecal occult blood test in a population-based colorectal cancer screening program. Br J Cancer 2014;111:21522155.

28. Rat C, Pogu C, Le Donné D, et al. Effect of physician notification regarding nonadherence to colorectal cancer screening on patient participation in fecal immunochemical test cancer screening: a randomized clinical trial. JAMA 2017;318:816-824.

29. Gupta S, Miller S, Koch M, et al. Financial incentives for promoting colorectal cancer screening: a randomized, comparative effec tiveness trial. Am J Gastroenterol 2016;111:1630-1636.

30. Hauptman N, Glavač D. Colorectal cancer blood-based biomarkers. Gastroenterol Res Pract 2017;2017:2195361.

31. Wu D, Zhou G, Jin P, et al. Detection of colorectal cancer using a simplified SEPT9 gene methylation assay is a reliable method for opportunistic screening. J Mol Diagn 2016;18:535-545. 
552 Gut and Liver, Vol. 14, No. 5, September 2020

32. Cohen JD, Li L, Wang Y, et al. Detection and localization of surgically resectable cancers with a multi-analyte blood test. Science 2018;359:926-930.
33. Murphy CC, Sigel BM, Yang E, et al. Adherence to colorectal cancer screening measured as the proportion of time covered. Gastrointest Endosc 2018;88:323-331. 